\title{
Topological Interactions in a Boltzmann-Type Framework
}

\author{
Adrien Blanchet $^{1}$. Pierre Degond $^{2}$
}

Received: 3 July 2015 / Accepted: 8 January 2016 / Published online: 17 February 2016

(C) The Author(s) 2016. This article is published with open access at Springerlink.com

\begin{abstract}
We consider a finite number of particles characterised by their positions and velocities. At random times a randomly chosen particle, the follower, adopts the velocity of another particle, the leader. The follower chooses its leader according to the proximity rank of the latter with respect to the former. We study the limit of a system size going to infinity and, under the assumption of propagation of chaos, show that the limit equation is akin to the Boltzmann equation. However, it exhibits a spatial non-locality instead of the classical non-locality in velocity space. This result relies on the approximation properties of Bernstein polynomials. We illustrate the dynamics with numerical simulations.
\end{abstract}

Keywords Rank · Topological interaction · Boltzmann equation

Mathematics Subject Classification $70 \mathrm{~K} 45 \cdot 92 \mathrm{D} 50 \cdot 91 \mathrm{C} 20$

\section{Introduction}

In this paper, we explore collective dynamics driven by rank-based interactions, i.e. that's to say interactions determined by the rank of the agents with respect to certain criterion. There are many examples where such interactions take place. In economics for instance, it was extensively analysed in [14] that agents are more sensitive to their rank compared to others (salary or wealth for example) than their own independent cardinal level. To go further, [17] studies, in an organisation, compensation schemes which pay according to an individual's ordinal rank rather than their output level. Such payoff based on the rank approach also

Pierre Degond

p.degond@imperial.ac.uk

Adrien Blanchet

Adrien.Blanchet@ut-capitole.fr

1 GREMAQ (IAST/TSE, UT1), 21 Allée de Brienne, 31000 Toulouse, France

2 Department of Mathematics, Imperial College London, London SW7 2AZ, UK 
appears very naturally in a variety of economics applications such as bids, the labour market, portfolio management, the oil market, academic production, reputation, etc.

Evolutionary game theory studies the evolution of strategies/genes transmitted through natural selection. In chimpanzees as in cockroaches a group is formed of a dominant male, females and lower order males. Only the dominant male is supposed to mate with the females. However, when the dominant male is absent, the females also reproduce with other males giving the preference to males in descending order [12]. It is also known that the rank of an offspring strongly depends on the rank of its mother [16], so that in the replicator dynamic process the rank increases the chance of reproduction. The study of such models requires taking into account interactions depending on the rank of the agents.

In this article we focus on the dynamics of bird flocks. There is a widespread literature of flocking models where the birds react to their neighbours as a function of the neighbours' distance from them within the flock. These are the co-called "metric" interactions. In this context, dynamics based on alignment [20], consensus [11] or attraction-repulsion see [3,4] have been widely studied. However, there has been recent compelling evidence [1] that interactions within bird flocks are mostly metric free, as the birds react primarily with a limited number of their nearest neighbours irrespective of the distances between them. This observation has motivated the concept of "topological interaction", which has been widely echoed in the scientific literature $[5,7,10,13,19]$.

Our goal is to investigate the large size limit of a system of agents interacting through topological interactions. Specifically, we consider a leader-follower model $[8,9]$ where at random times a randomly chosen bird, the follower, decides to adopt the velocity of another bird, its leader, in the flock. The follower chooses its leader according to a probability only depending on the proximity rank of the latter with respect to the former. If we assume that the probability has a strong cutoff as soon as the proximity rank exceeds a certain value, of the order of seven in actual flocks, the considered model is akin to the topological interactions of [1].

To our knowledge, [6] is the first mathematical work where interaction rules between agents depending on their rank are considered. The closest to our work is [15] where kinetic and hydrodynamic models for topological interactions have been proposed. However, the considered dynamics is different from ours. In [15], it is supposed that an agent's velocity relaxes towards an average velocity of its neighbours where the relative weights of the neighbours depend on their proximity rank to the considered agent. Therefore, it is a model of Cucker-Smale type [11] combined with a topological interaction rule. In [15], a mean-field type kinetic model is rigorously derived under some regularisation in the large system size limit and a hydrodynamic model under a monokinetic closure assumption is proposed.

Here, the interaction rule is different and, in the large system size limit, leads to a Boltzmann type model with an integral operator describing the balance between gains and losses due to the interactions rather than a mean-field model where the interactions are described through a force field. From the mathematical viewpoint, this makes a considerable difference, as an empirical measure approach is not possible. Instead, one has to rely on the propagation of chaos property for the solution of the master equation. In the present work, propagation of chaos is assumed and its proof is defered to future work. Still, under this assumption, the derivation of the kinetic equation is not obvious and as we will see, relies on fine approximation properties of Bernstein polynomials.

Indeed, we will realise that the derivation of a kinetic model requires the estimation of the probability that given two particles say numbered 1 and 2, the rank of 2 with respect to 1 be equal to a given integer $j$. Then, the interaction probability of 1 with 2 in this configuration is a function $K(j / N)$, where $N$ is the total number of particles and the function $K$ is characteristic of the considered interaction. Thanks to an easy combinatorial estimation, the total probability 
of 1 interacting with 2 is found as the Bernstein polynomial approximation of $K$ when $N$ is large. Due to some cancellations, the first order correction in powers of $1 / N$ of the Bernstein polynomial approximation of $K$ is also needed. This correction can be found in the literature [18].

The paper is organised as follows. In Sect. 2, we present the $N$ particle dynamics and state the main result. In Sect. 3, we derive the master equation of the process and the equation for the first marginal under the assumption of propagation of chaos. In Sect. 4 we precisely state our main result, namely that, in the limit $N \rightarrow \infty$ and the assumption of propagation of chaos, the equation for the first marginal reduces to a kinetic equation of Boltzmann type with spatial nonlinearity. To prove this theorem, we use results on Bernstein's polynomial approximation from the literature [18]. Section 5 offers some considerations on the limit kinetic equation and illustrates our discussion with numerical simulations emphasising that the large-time and large number of particles limits do not commute. Finally, a conclusion is drawn in Sect. 6.

\section{The $N$-particle Dynamics}

Consider a set of $N$ particles. The particle $i$ is characterised by its position $x_{i} \in \mathbb{R}^{n}$ and its velocity $v_{i} \in \mathbb{R}^{n}$ where $n \geq 1$ is both the spatial and velocity dimension. For a given particle $i$ we can order the other particles relatively to their distance to $i$. More precisely, we have the following:

Definition 1 (Rank) Consider $N$ particles located at $x_{1}, \ldots, x_{N}$. Consider the $i$-th particle and order the list $\left(\left|x_{j}-x_{i}\right|\right)_{j=1, \ldots, N, j \neq i}$ by increasing order and denote by $R^{N}(i, j) \in$ $\{1, \ldots, N-1\}$ the position of the $j$-th item in this list. If two indices $j$ and $j^{\prime}$ are such that $\left|x_{j}-x_{i}\right|=\left|x_{j^{\prime}}-x_{i}\right|$, then we choose arbitrarily an ordering between these two numbers. We define $R^{N}(i, i)=0$. Now, we define the rank of $j$ with respect to $i$ as:

$$
r^{N}(i, j)=\frac{R^{N}(i, j)}{N-1} \in \bigcup_{k=1}^{N-1}\left\{\frac{k}{N-1}\right\} .
$$

We introduce a function $K: r \in[0,1] \mapsto K(r) \in[0, \infty)$ such that

$$
\int_{0}^{1} K(r) \mathrm{d} r=1 \text {. }
$$

We define

$$
K^{N}(r)=\frac{K(r)}{\sum_{k=1}^{N-1} K\left(\frac{k}{N-1}\right)},
$$

in order to have for any $i \in\{1, \ldots, N\}$ :

$$
\sum_{\substack{j=1 \\ j \neq i}}^{N} K^{N}\left(r^{N}(i, j)\right)=\sum_{k=1}^{N-1} K^{N}\left(\frac{k}{N-1}\right)=1 .
$$

In this way, for any $i \in\{1, \ldots, N\}$, the collection $\left(\pi_{i j}\right)_{j=1, j \neq i}^{N}$, where

$$
\pi_{i j}^{N}=K^{N}\left(r^{N}(i, j)\right),
$$

defines a discrete probability measure on the set $\{j \in\{1, \ldots, N\}, j \neq i\}$. 
Consider $N$ particles $\left\{\left(x_{1}(t), v_{1}(t)\right), \ldots,\left(x_{N}(t), v_{N}(t)\right)\right\}$ which are subject to the following dynamics (previously referred to as the "Choose the Leader" dynamics $[8,9]$ ):

- The dynamics is a succession of free-flights and collisions.

- During free-flight, particles follow straight trajectories

$$
\left\{\begin{array}{c}
\dot{x}_{i}=v_{i} \\
\dot{v}_{i}=0
\end{array}\right.
$$

- At Poisson random times with a rate equals to $N$, particles undergo the following collisions process: Pick a particle $i$ in $\{1, \ldots, N\}$ with uniform probability $1 / N$ and perform a collision, i.e. pick a collision partner $j$ in the set $\{j \in\{1, \ldots, N\}, j \neq i\}$ with probability $\pi_{i j}^{N}$ and perform:

$$
\left\{\begin{array}{l}
\left(x_{i}, x_{j}\right) \text { remains unchanged, } \\
\left(v_{i}, v_{j}\right) \text { is changed into }\left(v_{j}, v_{j}\right) .
\end{array}\right.
$$

Remark 1 It is a priori unclear that the dynamics will be non-trivial in the limit $N \rightarrow \infty$ for a rate of the Poisson process equal to $N$. This will appear clearly in the section dealing with the passage to the limit $N \rightarrow \infty$ (see Sect. 4).

Since the rank of $j$ with respect to $i$ is an intrinsic property of the positions of the pair of particles and does not depend on how they are numbered, we have the following properties of the rank:

Remark 2 Let $\left(x_{1}, \ldots, x_{N}\right)$ be a set on $N$ particles.

(i) The rank $r^{N}(i, j)$, and hence $\pi_{i j}^{N}$, is a function of $\left(x_{1}, \ldots, x_{N}\right)$, i.e.

$$
r^{N}(i, j)=r^{N}(i, j)\left(x_{1}, \ldots, x_{N}\right) .
$$

More precisely, we consider the rank $r^{N}(i, j)$ as a function of $L^{\infty}\left(\mathbb{R}^{n N}\right)$.

(ii) The rank is permutation invariant, i.e. for any permutation $\sigma \in \mathfrak{S}_{N}$ where $\mathfrak{S}_{N}$ denotes the set of permutations of $\{1, \ldots, N\}$, we have

$$
r^{N}(\sigma(i), \sigma(j))\left(x_{\sigma(1)}, \ldots, x_{\sigma(N)}\right)=r^{N}(i, j)\left(x_{1}, \ldots, x_{N}\right) .
$$

The aim of this article is to study the limit of this dynamics when the number of particles goes to $\infty$. To do so we will assume that the propagation of chaos property holds true i.e.

$$
f^{(N)}\left(Z_{1}, \cdots, Z_{N}, t\right)=\prod_{\ell=1}^{N} f_{N}^{(1)}\left(Z_{\ell}, t\right), \quad \forall \mathbf{Z} \in \mathbb{R}^{2 n N}, \quad \forall t \in[0, \infty) .
$$

Assuming that $f_{N}^{(1)} \rightarrow f$ and $\rho_{N}^{(1)}:=\int f_{N}^{(1)} \mathrm{d} v \rightarrow \rho=\int f \mathrm{~d} v$, then in the limit $N \rightarrow \infty$, we will prove that $f$ is a solution of the kinetic equation:

$$
\frac{\partial f}{\partial t}(x, v)+v \cdot \nabla_{x} f(x, v)=\rho(x) \int f\left(x^{\prime}, v\right) K\left(M_{\rho}\left(x,\left|x^{\prime}-x\right|\right)\right) \mathrm{d} x^{\prime}-f(x, v),
$$

where $M_{\rho}$ is the partial mass of $\rho$ and is defined by

$$
M_{\rho}(x, s)=\int_{x^{\prime} \in B(x, s)} \rho\left(x^{\prime}\right) \mathrm{d} x^{\prime},
$$

and where $B(x, s)=\left\{y \in \mathbb{R}^{n}|| y-x \mid \leq s\right\}$ is the ball centred at $x$ and of radius $s>0$. 
Remark 3 The conservation of mass property holds true by Lemma 5 applied to $H=K$.

In the following section, we derive the master equation for this process, Sect. 3.1, and the first marginal equation for indistinguishable particles, Sect. 3.2. Then, in Sect. 3.3, we derive the master equation under the assumption of propagation of chaos.

\section{Master Equation and Propagation of Chaos}

\subsection{Master Equation}

To simplify the notation, when no confusion is possible, we will denote $\mathbf{x}:=\left(x_{1}, \ldots, x_{N}\right)$, $\mathbf{v}:=\left(v_{1}, \ldots, v_{N}\right), Z_{i}:=\left(x_{i}, v_{i}\right), \mathbf{Z}:=\left(Z_{1}, \ldots, Z_{N}\right)$ and $\mathrm{d} \mathbf{Z}:=\mathrm{d} x_{1} \mathrm{~d} v_{1} \ldots \mathrm{d} x_{N} \mathrm{~d} v_{N}$.

As the collisions occur at Poisson times with rate $N$, the master equation in weak form is, for all test function $\phi^{N}: \mathbf{Z} \mapsto \phi^{N}(\mathbf{Z})$ :

$$
\begin{aligned}
& \partial_{t} \int f^{(N)}(\mathbf{Z}) \phi^{N}(\mathbf{Z}) \mathrm{d} \mathbf{Z}-\sum_{i=1}^{N} \int f^{(N)}(\mathbf{Z})\left(v_{i} \cdot \nabla_{x_{i}}\right) \phi^{N}(\mathbf{Z}) \mathrm{d} \mathbf{Z} \\
& =N \int\left[\frac{1}{N} \sum_{\substack{i, j=1 \\
j \neq i}}^{N} \pi_{i j}^{N}(\mathbf{x}) \phi^{N}\left(Z_{1}, \ldots, x_{i}, v_{j}, \ldots, x_{j}, v_{j}, \ldots Z_{N}\right)-\phi^{N}(\mathbf{Z})\right] f^{(N)}(\mathbf{Z}) \mathrm{d} \mathbf{Z} \\
& =N \int\left[\frac{1}{N} \sum_{\substack{i, j=1 \\
j \neq i}}^{N} \int \pi_{i j}^{N}(\mathbf{x}) \phi^{N}\left(Z_{1}, \ldots, x_{i}, v_{i}^{\prime}, \ldots, x_{j}, v_{j}, \ldots Z_{N}\right) \delta\left(v_{i}^{\prime}-v_{j}\right) \mathrm{d} v_{i}^{\prime}\right. \\
& \left.-\phi^{N}(\mathbf{Z})\right] f^{(N)}(\mathbf{Z}) \mathrm{d} \mathbf{Z}
\end{aligned}
$$

By exchanging the notations $v_{i}$ and $v_{i}^{\prime}$ we obtain the following master equation in the strong form:

$$
\partial_{t} f^{(N)}(\mathbf{Z})=\sum_{i=1}^{N} f^{(N)}(\mathbf{Z})\left(v_{i} \cdot \nabla_{x_{i}}\right)+N L f^{(N)}(\mathbf{Z}),
$$

where the operator $L$ is defined by

$$
L f^{(N)}(\mathbf{Z}):=\frac{1}{N} \sum_{\substack{i, j=1 \\ i \neq j}}^{N} \pi_{i j}^{N}(\mathbf{x}) \delta\left(v_{i}-v_{j}\right) \int f^{(N)}\left(Z_{1}, \ldots, x_{i}, v_{i}^{\prime}, \ldots Z_{N}\right) \mathrm{d} v_{i}^{\prime}-f^{(N)}(\mathbf{Z}) .
$$

We now state a property which will be needed in the remainder of this article, namely that if $f^{(N)}(t)$ is permutation invariant at time $t=0$ then it stays permutation invariant for all times.

Lemma 1 (Invariance under permutation) Define for all $\sigma \in \mathfrak{S}_{N}$,

$$
\sigma f^{(N)}(\mathbf{Z}):=f^{(N)}\left(\mathbf{Z}_{\sigma(1)}, \ldots, \mathbf{Z}_{\sigma(N)}\right) .
$$


Then we have:

$$
L\left(\sigma f^{(N)}\right)=\sigma\left(L f^{(N)}\right) .
$$

As a consequence, if $f^{(N)}(t)$ is permutation invariant at time $t=0$, i.e. $\left.\sigma f^{(N)}(t)\right|_{t=0}=$ $\left.f^{(N)}(t)\right|_{t=0}$ for all $\sigma \in \mathfrak{S}_{N}$, then it is permutation invariant for all times.

Proof To emphasise the dependence in $\mathbf{Z}$, we can rewrite the operator $L$ as:

$$
L f^{(N)}(\mathbf{Z})=\frac{1}{N} \sum_{\substack{i, j=1 \\ i \neq j}}^{N} \pi_{i j}^{N}(\mathbf{x}(\mathbf{Z})) \delta\left(V_{i}(\mathbf{Z})-V_{j}(\mathbf{Z})\right) P_{i} f^{(N)}(\mathbf{Z})-f^{(N)}(\mathbf{Z}),
$$

with $\mathbf{x}(\mathbf{Z})=\mathbf{x}, V_{i}(\mathbf{Z})=v_{i}$ and

$$
P_{i} f^{(N)}(\mathbf{Z})=\int f^{(N)}\left(Z_{1}, \ldots, x_{i}, v_{i}^{\prime}, \ldots Z_{N}\right) \mathrm{d} v_{i}^{\prime} .
$$

First note that, setting $\sigma \mathbf{Z}=\left(Z_{\sigma(1)}, \ldots, Z_{\sigma(N)}\right)$, we have

$$
V_{i}(\mathbf{Z})=V_{\sigma(i)}(\sigma \mathbf{Z}), \text { and } P_{i}\left(\sigma f^{(N)}\right)(\mathbf{Z})=P_{\sigma(i)} f^{(N)}(\sigma \mathbf{Z}) .
$$

Therefore by applying the $\sigma^{-1}$ permutation to the double sum and using the permutation invariance of the rank, see Lemma 2 (ii), we obtain

$$
\begin{aligned}
& L \sigma f^{(N)}(\mathbf{Z})=\frac{1}{N} \sum_{\substack{i, j=1 \\
i \neq j}}^{N} \pi_{i j}^{N}(\mathbf{x}(\mathbf{Z})) \delta\left(V_{i}(\mathbf{Z})-V_{j}(\mathbf{Z})\right) P_{i}\left(\sigma f^{(N)}\right)(\mathbf{Z}) \\
& -\left(\sigma f^{(N)}\right)(\mathbf{Z}) \\
& =\frac{1}{N} \sum_{\substack{i, j=1 \\
i \neq j}}^{N} K^{N}\left[r^{N}(i, j)\right](\mathbf{x}(\mathbf{Z})) \delta\left(V_{i}(\mathbf{Z})-V_{j}(\mathbf{Z})\right) P_{\sigma(i)} f^{(N)}(\sigma \mathbf{Z}) \\
& -\left(\sigma f^{(N)}\right)(\mathbf{Z}) \\
& =\frac{1}{N} \sum_{\substack{i^{\prime}, j^{\prime}=1 \\
i^{\prime} \neq j^{\prime}}}^{N} K^{N}\left[r^{N}\left(\sigma^{-1}\left(i^{\prime}\right), \sigma^{-1}\left(j^{\prime}\right)\right)\right](\mathbf{x}(\mathbf{Z})) \delta\left(V_{\sigma^{-1}\left(i^{\prime}\right)}(\mathbf{Z})\right. \\
& \left.-V_{\sigma^{-1}\left(j^{\prime}\right)}(\mathbf{Z})\right) P_{i^{\prime}} f^{(N)}(\sigma \mathbf{Z})-\left(\sigma f^{(N)}\right)(\mathbf{Z}) \\
& =\frac{1}{N} \sum_{\substack{i^{\prime}, j^{\prime}=1 \\
i^{\prime} \neq j^{\prime}}}^{N} K^{N}\left[r^{N}\left(i^{\prime}, j^{\prime}\right)\right](\mathbf{x}(\sigma \mathbf{Z})) \delta\left(V_{i^{\prime}}(\sigma \mathbf{Z})\right. \\
& \left.-V_{j^{\prime}}(\sigma \mathbf{Z})\right) P_{i^{\prime}} f^{(N)}(\sigma \mathbf{Z})-f^{(N)}(\sigma \mathbf{Z}) \\
& =\left(L f^{(N)}\right)(\sigma \mathbf{Z})=\sigma L \partial_{t} f^{(N)}(\mathbf{Z}) .
\end{aligned}
$$

The above property states that $\sigma^{-1} L \sigma=L$, for all $\sigma \in \mathfrak{S}_{N}$. Supposing that $L$ is a bounded operator, we deduce that $\sigma^{-1} L^{k} \sigma=L^{k}$, for all $k \in \mathbb{N}$ and consequently $\sigma^{-1} e^{L} \sigma=e^{L}$. Now, the solution of the problem $\partial_{t} f^{(N)}=N L f^{(N)}$ with $\left.f^{(N)}\right|_{t=0}=f_{0}^{(N)}$ can be written $f^{(N)}(t)=e^{N L t} f_{0}^{(N)}$. We deduce that $\sigma f^{(N)}(t)=e^{N L t} \sigma f_{0}^{(N)}$. Therefore, if $\sigma f_{0}^{(N)}=f_{0}^{(N)}$, 
then $\sigma f^{(N)}(t)=f^{(N)}(t)$, for all $t \geq 0$. If $L$ is not bounded, the same property remains true thanks to an approximation argument.

\subsection{First Marginal Equation for Indistinguishable Particles}

In the remainder of this article, we will suppose that $f^{(N)}$ is invariant under permutations which physically means that the particles are indistinguishable. This allows us to define the $k$-particle marginal as

$$
f_{N}^{(k)}\left(Z_{1}, \ldots, Z_{k}, t\right)=\int f^{(N)}\left(Z_{1}, \ldots, Z_{N}, t\right) \mathrm{d} Z_{k+1} \ldots \mathrm{d} Z_{N}
$$

and $f_{N}^{(k)}$ is still invariant under permutations of $\left(Z_{1}, \ldots, Z_{k}\right)$.

Proposition 1 (First marginal equation for indistinguishable particles) Assume (A1). For any test functions satisfying

$$
\phi^{N}\left(Z_{1}, \ldots, Z_{N}\right)=\phi\left(Z_{1}\right)
$$

we have

$$
\begin{aligned}
\partial_{t} \int f_{N}^{(1)}\left(Z_{1}\right) \phi\left(Z_{1}\right) \mathrm{d} Z_{1}= & \sum_{i=1}^{N} \int f_{N}^{(1)}(\mathbf{Z})\left(v_{i} \cdot \nabla_{x_{i}}\right) \phi(\mathbf{Z}) \mathrm{d} \mathbf{Z} \\
& +(N-1) \int \pi_{12}^{N}(\mathbf{x}) \phi\left(x_{1}, v_{2}\right) f^{(N)}(\mathbf{Z}) \mathrm{d} \mathbf{Z} \\
& +(N-1) \int \pi_{21}^{N}(\mathbf{x}) \phi\left(Z_{1}\right) f^{(N)}(\mathbf{Z}) \mathrm{d} \mathbf{Z} \\
& +(N-1)(N-2) \int \pi_{23}^{N}(\mathbf{x}) \phi\left(Z_{1}\right) f^{(N)}(\mathbf{Z}) \mathrm{d} \mathbf{Z} \\
& -N \int \phi\left(Z_{1}\right) f_{N}^{(1)}\left(Z_{1}\right) \mathrm{d} Z_{1} .
\end{aligned}
$$

Remark 4 Since $\pi_{i j}^{N}=K^{N}\left(r^{N}(i, j)\right)$ and $K^{N}(r)=K(r) / \sum K(k /(N-1)), \pi_{i j}^{N}$ is of order $1 / N$. Hence the first three terms are of order 1 while the last two ones are of order $N$. Section 4 will be devoted to the study of these terms and to the proof that the difference of the last two terms is of the same order as the first three ones.

Proof Separating the cases $i=1 \neq j, j=1 \neq i$, and $i \geq 2, j \geq 2$, the master equation (1) gives

$$
\begin{aligned}
& \partial_{t} \int f^{(N)}(\mathbf{Z}) \phi(\mathbf{Z}) \mathrm{d} \mathbf{Z} \\
& =\sum_{j=2}^{N} A_{j}^{(1)}+\sum_{i=2}^{N} A_{i}^{(2)}+\sum_{i=2}^{N} \sum_{j=2, j \neq i}^{N} A_{i, j}-N \int \phi\left(x_{1}, v_{1}\right) f^{(N)}(\mathbf{Z}) \mathrm{d} \mathbf{Z},
\end{aligned}
$$

with

$$
\begin{aligned}
A_{j}^{(1)} & :=\int \pi_{1 j}^{N}(\mathbf{x}) \phi\left(x_{1}, v_{j}\right) f^{(N)}(\mathbf{Z}) \mathrm{d} \mathbf{Z}, \\
A_{i}^{(2)} & :=\int \pi_{i 1}^{N}(\mathbf{x}) \phi\left(Z_{1}\right) f^{(N)}(\mathbf{Z}) \mathrm{d} \mathbf{Z}, \\
A_{i, j} & :=\int \pi_{i j}^{N}(\mathbf{x}) \phi\left(Z_{1}\right) f^{(N)}(\mathbf{Z}) \mathrm{d} \mathbf{Z} .
\end{aligned}
$$


To compute the first term $A_{j}^{(1)}$, we perform the change of variables $Z_{2}^{\prime}=Z_{j}$ and $Z_{j}^{\prime}=Z_{2}$, which leads to:

$$
\begin{aligned}
A_{j}^{(1)}= & \int \pi_{1 j}^{N}\left(x_{1}, x_{j}^{\prime}, \ldots, x_{2}^{\prime}, \ldots, x_{N}\right) \phi\left(x_{1}, v_{2}^{\prime}\right) f^{(N)}\left(Z_{1}, Z_{j}^{\prime}, \ldots, Z_{2}^{\prime}, \ldots, Z_{N}\right) \\
& \times \mathrm{d} Z_{1} \mathrm{~d} Z_{j}^{\prime} \ldots \mathrm{d} Z_{2}^{\prime} \ldots \mathrm{d} Z_{N} .
\end{aligned}
$$

Using the permutation invariance of the rank (see Lemma 2 (ii)), we have

$$
\pi_{1 j}^{N}\left(x_{1}, x_{j}^{\prime}, \ldots, x_{2}^{\prime}, \ldots, x_{N}\right)=\pi_{12}^{N}\left(x_{1}, x_{2}^{\prime}, \ldots, x_{j}^{\prime}, \ldots, x_{N}\right) .
$$

Therefore, dropping the primes and using the permutation invariance of $f^{(N)}$, we obtain

$$
A_{j}^{(1)}=\int \pi_{12}^{N}(\mathbf{x}) \phi\left(x_{1}, v_{2}\right) f^{(N)}(\mathbf{Z}) \mathrm{d} \mathbf{Z},
$$

which does not depend on $j$.

Similarly, we have

$$
\begin{aligned}
A_{i}^{(2)}= & \int \pi_{i 1}^{N}\left(x_{1}, x_{i}, \ldots, x_{2}, \ldots, x_{N}\right) \phi\left(Z_{1}\right) f^{(N)}\left(Z_{1}, Z_{i}, \ldots, Z_{2}, \ldots, Z_{N}\right) \\
& \times \mathrm{d} Z_{1} \mathrm{~d} Z_{i} \ldots \mathrm{d} Z_{2} \ldots \mathrm{d} Z_{N}
\end{aligned}
$$

so that, using the permutation invariance of the rank, see Lemma 2 (ii), and the permutation invariance of $f^{(N)}$ as previously, we obtain

$$
A_{i}^{(2)}=\int \pi_{21}^{N}(\mathbf{x}) \phi\left(Z_{1}\right) f^{(N)}(\mathbf{Z}) \mathrm{d} \mathbf{Z},
$$

which does not depend on $i$.

Also, we have with $i \geq 2, j \geq 2$ and $i \neq j$ :

$$
\begin{aligned}
A_{i, j}= & \int \pi_{i j}^{N}\left(x_{1}, x_{i}, x_{j} \ldots, x_{2}, \ldots, x_{3}, \ldots, x_{N}\right) \phi\left(Z_{1}\right) \\
& \times f^{(N)}\left(Z_{1}, Z_{i}, Z_{j}, \ldots, Z_{2}, \ldots, Z_{3}, \ldots Z_{N}\right) \\
& \times \mathrm{d} Z_{1} \mathrm{~d} Z_{i} \mathrm{~d} Z_{j} \ldots \mathrm{d} Z_{2} \ldots \mathrm{d} Z_{3} \ldots \mathrm{d} Z_{N} .
\end{aligned}
$$

Then using the permutation invariance of the rank, see Lemma 2 (ii), and the permutation invariance of $f^{(N)}$ as previously, we obtain

$$
A_{i, j}=\int \pi_{23}^{N}(\mathbf{x}) \phi\left(Z_{1}\right) f^{(N)}(\mathbf{Z}) \mathrm{d} \mathbf{Z} .
$$

For the last term of (3) we obviously have

$$
\int \phi\left(Z_{1}\right) f^{(N)}(\mathbf{Z}) \mathrm{d} \mathbf{Z}=\int \phi\left(Z_{1}\right) f_{N}^{(1)}\left(Z_{1}\right) \mathrm{d} Z_{1} .
$$

Collecting all these identities, we obtain the identity stated in Proposition 1.

\subsection{Propagation of Chaos}

Assume now that the propagation of chaos property holds true i.e.

$$
f^{(N)}\left(Z_{1}, \cdots, Z_{N}, t\right)=\prod_{\ell=1}^{N} f_{N}^{(1)}\left(Z_{\ell}, t\right), \quad \forall \mathbf{Z} \in \mathbb{R}^{2 n N}, \quad \forall t \in[0, \infty),
$$


and define:

$$
\rho_{N}^{(1)}(x)=\int f_{N}^{(1)}(x, v) \mathrm{d} v
$$

We remark that $\rho_{N}^{(1)}$ is a probability density.

We have the following proposition:

Proposition 2 (First marginal equation with propagation of chaos) Assume (A2). For any test functions satisfying (2), we have

$$
\begin{aligned}
\partial_{t} & \int f_{N}^{(1)}\left(Z_{1}\right) \phi\left(Z_{1}\right) \mathrm{d} Z_{1} \\
& =\sum_{i=1}^{N} \int f_{N}^{(1)}(\mathbf{Z})\left(v_{i} \cdot \nabla_{x_{i}}\right) \phi(\mathbf{Z}) \mathrm{d} \mathbf{Z}+\left(A^{N}\right)+\left(B^{N}\right)+\left(C^{N}\right)+\left(D^{N}\right),
\end{aligned}
$$

with

$$
\begin{aligned}
\left(A^{N}\right)= & \frac{1}{S^{N}(K)} \int \phi\left(x_{1}, v_{2}\right) f_{N}^{(1)}\left(Z_{1}\right) f_{N}^{(1)}\left(Z_{2}\right) \\
& \times K\left(r^{N}(1,2)(\mathbf{x})\right) \prod_{\ell=3}^{N} \rho_{N}^{(1)}\left(x_{\ell}\right) \mathrm{d} x_{\ell} \mathrm{d} Z_{1} \mathrm{~d} Z_{2}, \\
\left(B^{N}\right)= & \frac{1}{S^{N}(K)} \int \phi\left(Z_{1}\right) f_{N}^{(1)}\left(Z_{1}\right) K\left(r^{N}(2,1)(\mathbf{x})\right) \prod_{\ell=2}^{N} \rho_{N}^{(1)}\left(x_{\ell}\right) \mathrm{d} x_{\ell} \mathrm{d} Z_{1}, \\
\left(C^{N}\right)= & \frac{N-2}{S^{N}(K)} \int \phi\left(Z_{1}\right) f_{N}^{(1)}\left(Z_{1}\right) K\left(r^{N}(2,3)(\mathbf{x})\right) \prod_{\ell=2}^{N} \rho_{N}^{(1)}\left(x_{\ell}\right) \mathrm{d} x_{\ell} \mathrm{d} Z_{1}, \\
\left(D^{N}\right)= & -N \int \phi\left(Z_{1}\right) f_{N}^{(1)}\left(Z_{1}\right) \mathrm{d} Z_{1},
\end{aligned}
$$

where $S^{N}(K)$ is given by

$$
S^{N}(K)=\frac{1}{N-1} \sum_{k=1}^{N-1} K\left(\frac{k}{N-1}\right) .
$$

We note that $S^{N}(K)$ is the Riemann sum approximation of $\int_{0}^{1} K(r) \mathrm{d} r$. Since we assume $\int_{0}^{1} K(r) \mathrm{d} r=1, S^{N}(K)$ converges to 1 as $N$ goes to $\infty$. Hence $\left(A^{N}\right)$ and $\left(B^{N}\right)$ are of order 1 , while $\left(C^{N}\right)$ and $\left(D^{N}\right)$ are of order $N$. In the next section we will prove that the difference $\left(C^{N}\right)+\left(D^{N}\right)$ is actually of order 1.

Proof This result is a direct consequence of Proposition 1, integrating in $v$ when possible. We then use that

$$
K^{N}=\frac{K}{(N-1) S_{N}(K)},
$$

to obtain the stated result. 


\section{Limit Equation}

For a density $\rho$, define the partial mass of $\rho$ centred in $x$ and of radius $s$ by:

$$
M_{\rho}(x, s)=\int_{\left|x-x^{\prime}\right| \leq s} \rho\left(x^{\prime}\right) \mathrm{d} x^{\prime} .
$$

We now state the main theorem of this article:

Theorem 1 (Limit equation) Assume (2). If

$$
\lim _{N \rightarrow \infty} f_{N}^{(1)} \rightarrow f \text { and } \lim _{N \rightarrow \infty} \rho_{N}^{(1)} \rightarrow \rho=\int f \mathrm{~d} v
$$

then, in the limit $N \rightarrow \infty$, for all test functions $\phi$ we have:

$$
\begin{aligned}
\partial_{t} & \int f(\mathbf{Z}) \phi(\mathbf{Z}) \mathrm{d} \mathbf{Z} \\
= & \int \phi\left(x_{1}, v_{2}\right) f\left(Z_{2}\right) \rho\left(x_{1}\right) K\left(M_{\rho}\left(x_{1},\left|x_{2}-x_{1}\right|\right) \mathrm{d} x_{1} \mathrm{~d} Z_{2}\right. \\
& -\int \phi\left(Z_{1}\right) f\left(Z_{1}\right) \mathrm{d} Z_{1},
\end{aligned}
$$

or, in strong form:

$$
\frac{\partial f}{\partial t}(x, v)+v \cdot \nabla_{x} f(x, v)=\rho(x) \int f\left(x^{\prime}, v\right) K\left(M_{\rho}\left(x,\left|x^{\prime}-x\right|\right)\right) \mathrm{d} x^{\prime}-f(x, v) .
$$

This result will be obtained by passing to the limit when $N \rightarrow \infty$ in (4). To pass to the limit in the transport term of (4) is classical and we refer the reader to classical textbooks on the subject. We divide the proof of this theorem in two sections. Section 4.1 is dedicated to the analysis of the terms $\left(A^{N}\right)$ and $\left(B^{N}\right)$ of (4). We prove that in the limit these two terms are of order 1 and can be expressed by means of the partial mass (see Proposition 4). The proofs rely on a combinatorial interpretation of the rank and on the fact that the integrals can be seen as the expectation of a suitable random variable (see Lemma 2). In Sect. 4.2 we use the same idea of interpreting the integrals occurring in the expression of $\left(C^{N}\right)$ as the expectation of a suitable random variable: the integral is dealt with in Lemma 3 and the sum $\left(C^{N}\right)+\left(D^{N}\right)$ will be proved to be of order 1 as stated in Proposition 5.

The main common difficulty while using the expectation interpretation of these terms is to evaluate truncated binomial sums. To handle this computation, the proofs will make an intensive and crucial use of the Bernstein polynomial approximation:

Proposition 3 (Bernstein polynomial approximation, [18]) Let $f$ be a function defined on $[0,1]$. The $n$-th Bernstein polynomial associated with $f$ is defined by

$$
B_{n}(f ; x):=\sum_{i=0}^{n} f\left(\frac{i}{n}\right)\left(\begin{array}{l}
n \\
i
\end{array}\right) x^{i}(1-x)^{n-i} .
$$

If $f \in \mathcal{C}^{2}[0,1]$ then

$$
B_{n}(f ; x)=f(x)+\frac{x(1-x)}{2 n} f^{\prime \prime}(x)+o\left(\frac{1}{n}\right) .
$$




\subsection{Evaluation of $\left(A^{N}\right)$ and $\left(B^{N}\right)$}

We first express the integrals involved in $\left(A^{N}\right)$ and $\left(B^{N}\right)$ in terms of the partial mass and determine the leading order behaviour of these quantities when $N$ is large:

Proposition 4 (Evaluation of $\left(A^{N}\right)$ and $\left(B^{N}\right)$ ) Under the assumptions of Theorem 1, we have for large $N$

$$
\begin{aligned}
& S^{N}(K) \times\left(A^{N}\right) \\
& \quad=\int \phi\left(x_{1}, v_{2}\right) \rho_{N}^{(1)}\left(Z_{1}\right) f_{N}^{(1)}\left(Z_{2}\right) K\left(M_{\rho_{N}^{(1)}}\left(x_{1},\left|x_{1}-x_{2}\right|\right)\right) \mathrm{d} x_{1} \mathrm{~d} Z_{2}+o(1),
\end{aligned}
$$

and

$$
\begin{aligned}
& S^{N}(K) \times\left(B^{N}\right) \\
& \quad=\int \phi\left(Z_{1}\right) f_{N}^{(1)}\left(Z_{1}\right) K\left(M_{\rho_{N}^{(1)}}\left(x_{2},\left|x_{1}-x_{2}\right|\right)\right) \rho_{N}^{(1)}\left(x_{2}\right) \mathrm{d} Z_{1} \mathrm{~d} x_{2}+o(1) .
\end{aligned}
$$

To prove this result we first interpret the integrals involved in $\left(A^{N}\right)$ and $\left(B^{N}\right)$ as expectations of suitable random variables. We first state the

Lemma 2 Under the assumptions of Theorem 1, we have for $N$ large,

$$
\int K\left(r^{N}(1,2)(\mathbf{x})\right) \prod_{\ell=3}^{N} \rho_{N}^{(1)}\left(x_{\ell}\right) \mathrm{d} x_{\ell}=K\left(M_{\rho_{N}^{(1)}}\left(x_{1},\left|x_{1}-x_{2}\right|\right)\right)+o(1),
$$

and

$$
\begin{aligned}
& \int K\left(r^{N}(2,1)(\mathbf{x})\right) \prod_{\ell=2}^{N} \rho_{N}^{(1)}\left(x_{\ell}\right) \mathrm{d} x_{\ell} \\
& =\int K\left(M_{\rho_{N}^{(1)}}\left(x_{2},\left|x_{1}-x_{2}\right|\right)\right) \rho_{N}^{(1)}\left(x_{2}\right) \mathrm{d} x_{2}+o(1) .
\end{aligned}
$$

Proof We first give a combinatorial interpretation of the rank and then use it to interpret the various expressions involved in the statement of the Lemma as expectations over suitably chosen random variables.

- Let us fix $x_{1}$ and $x_{2}$. The $\operatorname{rank} r^{N}(1,2)$ is equal to the number of points $x_{3}, \ldots, x_{N}$ belonging to the ball $B=B\left(x_{1},\left|x_{2}-x_{1}\right|\right)=\left\{x:\left|x-x_{1}\right| \leq\left|x_{2}-x_{1}\right|\right\}$ plus one unit, scaled by the factor $N-1$, i.e.

$$
r^{N}(1,2)(\mathbf{x})=\frac{\#\left\{j \in\{3, \ldots, N\}: x_{j} \in B\right\}+1}{N-1} .
$$

Denote $P_{R}$ be the probability such that $R^{N}(1,2)=R$ where $R^{N}(1,2)=(N-1) r^{N}(1,2)$. To have $R^{N}(1,2)=R$, we have to choose $R-1$ particles amongst $N-2$ to lie in $B$. The probability that one of the $R-1$ particles belongs to $B$ is equal to

$$
p:=M_{\rho_{N}^{(1)}}\left(x_{1},\left|x_{2}-x_{1}\right|\right),
$$

while the probability that one of the $N-2-(R-1)$ remaining particles lies in $\mathbb{R}^{n} \backslash B$ is $1-p$. Therefore,

$$
P_{R}=\left(\begin{array}{l}
N-2 \\
R-1
\end{array}\right) p^{R-1}(1-p)^{N-2-(R-1)}
$$


- Now, $x_{1}$ and $x_{2}$ being fixed, the quantity

$$
\int K\left(r^{N}(1,2)(\mathbf{x})\right) \prod_{\ell=3}^{N} \rho_{N}^{(1)}\left(x_{\ell}\right) \mathrm{d} x_{\ell}
$$

can be interpreted as the expectation of $K\left(r^{N}(1,2)\right)$ when $N-2$ points $x_{3}, \ldots, x_{N}$ are drawn according to independent identically distributed probabilities with density $\rho_{N}^{(1)}(x) \mathrm{d} x$. It will be denoted $\mathbb{E}\left\{K\left(r^{N}(1,2)(\mathbf{x})\right)\right\}$.

By (5), we compute

$$
\begin{aligned}
\mathbb{E}\left\{K\left(r^{N}(1,2)(\mathbf{x})\right)\right\} & =\sum_{R=1}^{N-1} K\left(\frac{R}{N-1}\right)\left(\begin{array}{l}
N-2 \\
R-1
\end{array}\right) p^{R-1}(1-p)^{N-2-(R-1)} \\
& =\sum_{R=0}^{M} K\left(\frac{R+1}{M+1}\right)\left(\begin{array}{l}
M \\
R
\end{array}\right) p^{R}(1-p)^{M-R}
\end{aligned}
$$

with $M=N-2$. Since, for $N$ large, $K((R+1) /(M+1))=K(R / M)+o(1)$ (remarking that $R / M \leq 1$ ),

$$
\mathbb{E}\left\{K\left(r^{N}(1,2)(\mathbf{x})\right)\right\}=\sum_{R=0}^{M} K\left(\frac{R}{M}\right)\left(\begin{array}{c}
M \\
R
\end{array}\right) p^{R}(1-p)^{M-R}+o(1) .
$$

Using Bernstein's approximation, Proposition 3, we obtain

$$
\mathbb{E}\left\{K\left(r^{N}(1,2)(\mathbf{x})\right)\right\}=K(p)+o(1) .
$$

Which is the first statement.

- The identity

$$
\int K\left(r^{N}(2,1)(\mathbf{x})\right) \prod_{\ell=3}^{N} \rho_{N}^{(1)}\left(x_{\ell}\right) \mathrm{d} x_{\ell}=K\left(M_{\rho_{N}^{(1)}}\left(x_{1},\left|x_{2}-x_{1}\right|\right)\right)+o(1),
$$

is obtained in an analogous way by exchanging the role of 1 and 2 . We then have to integrate by $\rho_{N}^{(1)}\left(x_{2}\right) \mathrm{d} x_{2}$ to obtain the stated result.

Proof of Proposition 4 Inserting the expressions of Lemma 2 in $\left(A^{N}\right)$ and $\left(B^{N}\right)$ we readily obtain the stated result.

\subsection{Evaluation of $\left(C^{N}\right)+\left(D^{N}\right)$}

Similarly to the previous section, the aim of this section is to determine the leading order behaviour of $\left(C^{N}\right)+\left(D^{N}\right)$ when $N$ is large:

Proposition 5 (Evaluation of $\left.\left(C^{N}\right)+\left(D^{N}\right)\right)$ Under the assumptions of Theorem 1, we have

$$
\begin{aligned}
\left(C^{N}\right)+\left(D^{N}\right)= & -\int \phi\left(Z_{1}\right) f_{N}^{(1)}\left(Z_{1}\right) \mathrm{d} Z_{1} \\
& -\int \phi\left(Z_{1}\right) f_{N}^{(1)}\left(Z_{1}\right) \rho_{N}^{(1)}\left(x_{2}\right) K\left(M_{\rho_{N}^{(1)}}\left(x_{2},\left|x_{1}-x_{2}\right|\right) \mathrm{d} x_{2} \mathrm{~d} Z_{1}+o(1) .\right.
\end{aligned}
$$


Like in the previous section, the first step of the proof consists of interpreting some of the integrals involved in $\left(C^{N}\right)$ as an expectation over a suitable random variable (see Lemma 3) and then the remaining integral (see Lemma 4). The proof of Proposition 5 will then just be a collection of these estimates with terms coming from $\int K(s) \mathrm{d} s$.

Lemma 3 Under the assumptions of Theorem 1, we have for large $N$

$$
\begin{aligned}
\int & K\left(r^{N}(2,3)(\mathbf{x})\right) \prod_{\ell=4}^{N} \rho_{N}^{(1)}\left(x_{\ell}\right) \mathrm{d} x_{\ell} \\
= & K\left(p_{23}\right)-\frac{K^{\prime}\left(p_{23}\right)}{N}\left(1-\chi_{B\left(x_{2},\left|x_{2}-x_{3}\right|\right)}\left(x_{1}\right)\right) \\
& +\frac{1}{N}\left[\frac{p_{23}\left(1-p_{23}\right)}{2} K^{\prime \prime}\left(p_{23}\right)+2\left(1-p_{23}\right) K^{\prime}\left(p_{23}\right)\right]+o\left(\frac{1}{N}\right),
\end{aligned}
$$

where $p_{23}=M_{\rho_{N}^{(1)}}\left(x_{2},\left|x_{2}-x_{3}\right|\right)$ only depends on $x_{2}$ and $x_{3}$ and

$$
\chi_{B\left(x_{2},\left|x_{2}-x_{3}\right|\right)}\left(x_{1}\right)= \begin{cases}1 & \text { if } x_{1} \in B\left(x_{2},\left|x_{2}-x_{3}\right|\right) \\ 0 & \text { otherwise }\end{cases}
$$

Proof Similarly to the proof of Lemma 2, we interpret the quantity

$$
\int K\left(r^{N}(2,3)(\mathbf{x})\right) \prod_{\ell=4}^{N} \rho_{N}^{(1)}\left(x_{\ell}\right) \mathrm{d} x_{\ell},
$$

as the expectation of $K\left(r^{N}(2,3)(\mathbf{x})\right)$ when the $N-4$ points $x_{4}, \ldots, x_{N}$ are drawn according to independent identically distributed probabilities with density $\rho_{N}^{(1)}(x) \mathrm{d} x$. Two cases have to be distinguished:

- First case: if $x_{1} \in B\left(x_{2},\left|x_{2}-x_{3}\right|\right)$ - Like in the proof of Lemma 2 we have

$$
r^{N}(2,3)=\frac{\#\left\{j \in\{4 \ldots, N\} \mid: x_{j} \in B\left(x_{2},\left|x_{2}-x_{3}\right|\right)\right\}+2}{N-1} .
$$

Hence, setting $p_{23}=M_{\rho_{N}^{(1)}}\left(x_{2},\left|x_{2}-x_{3}\right|\right)=: p$,

$$
\begin{aligned}
\mathbb{E}\left\{K\left(r^{N}(2,3)(\mathbf{x})\right)\right\} & =\sum_{R=2}^{N-1} K\left(\frac{R}{N-1}\right)\left(\begin{array}{l}
N-3 \\
R-2
\end{array}\right) p^{R-2}(1-p)^{N-3-(R-2)} \\
& =\sum_{R=0}^{M} K\left(\frac{R+2}{M+2}\right)\left(\begin{array}{c}
M \\
R
\end{array}\right) p^{R}(1-p)^{M-R}
\end{aligned}
$$

with $M=N-3$. By expanding $K$, we have, uniformly with respect to $R \in\{0, \cdots, M\}$

$$
K\left(\frac{R+2}{M+2}\right)=K\left(\frac{R}{M}\right)+\frac{2}{M}\left(\frac{M-R}{M+2}\right) K^{\prime}\left(\frac{R}{M}\right)+o\left(\frac{1}{M}\right) .
$$

Since $(M-R) /(M+2)=1-M / R+o(1)$ we obtain uniformly with respect to $R \in$ $\{0, \cdots, M\}$

$$
K\left(\frac{R+2}{M+2}\right)=K\left(\frac{R}{M}\right)+\frac{2}{M}\left(1-\frac{R}{M}\right) K^{\prime}\left(\frac{R}{M}\right)+o\left(\frac{1}{M}\right) .
$$


So, we obtain

$$
\begin{aligned}
\mathbb{E}\left\{K\left(r^{N}(2,3)(\mathbf{x})\right)\right\}= & \sum_{R=0}^{M} K\left(\frac{R}{M}\right)\left(\begin{array}{c}
M \\
R
\end{array}\right) p^{R}(1-p)^{M-R} \\
& +\frac{2}{M} \sum_{R=0}^{M}\left(1-\frac{R}{M}\right) K^{\prime}\left(\frac{R}{M}\right)\left(\begin{array}{c}
M \\
R
\end{array}\right) p^{R}(1-p)^{M-R}+o(1) .
\end{aligned}
$$

Using Bernstein's approximation, Proposition 3, to $K$ and to $p \mapsto(1-p) K^{\prime}(p)$ we obtain

$$
\begin{aligned}
\mathbb{E}\left\{K\left(r^{N}(2,3)(\mathbf{x})\right)\right\} & =K(p)+\frac{p(1-p)}{2 M} K^{\prime \prime}(p)+\frac{2(1-p)}{M} K^{\prime}(p)+o(1) \\
& =K(p)+\frac{2(1-p)}{N} K^{\prime}(p)+\frac{p(1-p)}{2 N} K^{\prime \prime}(p)+o(1) .
\end{aligned}
$$

- Second case: if $x_{1} \notin B\left(x_{2},\left|x_{2}-x_{3}\right|\right)$ - In this case,

$$
r^{N}(2,3)=\frac{\#\left\{j \in\{4 \ldots, N\}: x_{j} \in B\left(x_{2},\left|x_{2}-x_{3}\right|\right)\right\}+1}{N-1} .
$$

Following the same step as before we compute, with $p=M_{\rho_{N}^{(1)}}\left(x_{2},\left|x_{2}-x_{3}\right|\right)$

$$
\mathbb{E}\left\{K\left(r^{N}(2,3)(\mathbf{x})\right)\right\}=\sum_{R=1}^{N-2} K\left(\frac{R}{N-1}\right)\left(\begin{array}{l}
N-3 \\
R-1
\end{array}\right) p^{R-1}(1-p)^{N-3-(R-1)},
$$

which we rewrite

$$
\mathbb{E}\left\{\left(r^{N}(2,3)(\mathbf{x})\right)\right\}=\sum_{R=0}^{M} K\left(\frac{R+1}{M+2}\right)\left(\begin{array}{c}
M \\
R
\end{array}\right) p^{R}(1-p)^{M-R},
$$

with $M=N-3$. By expanding $K$, we have

$$
K\left(\frac{R+1}{M+2}\right)=K\left(\frac{R}{M}\right)+\frac{1}{M}\left(1-\frac{2 R}{M}\right) K^{\prime}\left(\frac{R}{M}\right)+o\left(\frac{1}{M}\right) .
$$

So, we have

$$
\begin{aligned}
\mathbb{E}\left\{\left(r^{N}(2,3)(\mathbf{x})\right)\right\}= & \sum_{R=0}^{M} K\left(\frac{R}{M}\right)\left(\begin{array}{c}
M \\
R
\end{array}\right) p^{R}(1-p)^{M-R} \\
& +\frac{1}{M} \sum_{R=0}^{M}\left(1-\frac{2 R}{M}\right) K^{\prime}\left(\frac{R}{M}\right)\left(\begin{array}{c}
M \\
R
\end{array}\right) p^{R}(1-p)^{M-R}+o(1) .
\end{aligned}
$$

Using Bernstein's approximation (see Proposition 3), we obtain

$$
\begin{aligned}
\mathbb{E}\left\{\left(r^{N}(2,3)(\mathbf{x})\right)\right\} & =K(p)+\frac{p(1-p)}{2 M} K^{\prime \prime}(p)+\frac{1-2 p}{M} K^{\prime}(p)+o(1) \\
& =K(p)+\frac{2(1-p)}{N} K^{\prime}(p)-\frac{K^{\prime}(p)}{N}+\frac{p(1-p)}{2 N} K^{\prime \prime}(p)+o(1) .
\end{aligned}
$$

- We obtain the result stated in Lemma 3 by noticing that Expression (7) is equal to the sum of Expression (6) and an extra term $-K^{\prime}(p) / N$. 
Lemma 4 (Evaluation of $S^{N}(K) \times\left(C^{N}\right) /(N-2)$ ) Under the assumptions of Theorem 1 , we have

$$
\begin{aligned}
\frac{S^{N}(K)}{N-2} \times\left(C^{N}\right)= & \left(1+\frac{1}{N}+\frac{K(1)-K(0)}{2 N}\right) \int \phi\left(Z_{1}\right) f_{N}^{(1)}\left(Z_{1}\right) \mathrm{d} Z_{1} \\
& -\frac{1}{N} \int \phi\left(Z_{1}\right) f_{N}^{(1)}\left(Z_{1}\right) \rho_{N}^{(1)}\left(x_{2}\right) K\left(M_{\rho_{N}^{(1)}}\left(x_{2},\left|x_{2}-x_{3}\right|\right) \mathrm{d} x_{2} \mathrm{~d} Z_{1}+o(1) .\right.
\end{aligned}
$$

Proof Using Lemma 3 and separating the cases $x_{1} \in B\left(x_{2},\left|x_{2}-x_{3}\right|\right)$ and $x_{1} \notin B\left(x_{2}, \mid x_{2}-\right.$ $x_{3} \mid$ ), we can write

$$
\frac{S^{N}(K)}{N-2} \times\left(C^{N}\right)=(1)+(2)+o(1),
$$

where, writing $p$ for $p_{23}$, i.e. $p=M_{\rho_{N}^{(1)}}\left(x_{2},\left|x_{2}-x_{3}\right|\right)$, we have:

$$
\begin{aligned}
(1)= & \frac{1}{N} \int_{x_{1} \in B\left(x_{2},\left|x_{2}-x_{3}\right|\right)} \phi\left(Z_{1}\right) f_{N}^{(1)}\left(Z_{1}\right) K^{\prime}(p) \rho_{N}^{(1)}\left(x_{2}\right) \rho_{N}^{(1)}\left(x_{3}\right) \mathrm{d} x_{2} \mathrm{~d} x_{3} \mathrm{~d} Z_{1} \\
& +o\left(\frac{1}{N}\right)
\end{aligned}
$$

and

$$
\begin{aligned}
(2)= & \int \phi\left(Z_{1}\right) f_{N}^{(1)}\left(Z_{1}\right)\left(K(p)+\frac{1-2 p}{N} K^{\prime}(p)+\frac{p(1-p)}{2 N} K^{\prime \prime}(p)\right) \\
& \times \rho_{N}^{(1)}\left(x_{2}\right) \rho_{N}^{(1)}\left(x_{3}\right) \mathrm{d} x_{2} \mathrm{~d} x_{3} \mathrm{~d} Z_{1}+o\left(\frac{1}{N}\right) .
\end{aligned}
$$

- For the term (1), we first notice that $x_{1} \in B\left(x_{2},\left|x_{2}-x_{3}\right|\right)$ is equivalent to saying that $x_{3} \notin B\left(x_{2},\left|x_{1}-x_{2}\right|\right)$ so that, for $p=M_{\rho_{N}^{(1)}}\left(x_{2},\left|x_{2}-x_{3}\right|\right)$,

$$
\begin{aligned}
(1)= & \frac{1}{N} \int_{x_{3} \notin B\left(x_{2},\left|x_{1}-x_{2}\right|\right)} \phi\left(Z_{1}\right) f_{N}^{(1)}\left(Z_{1}\right) K^{\prime}(p) \rho_{N}^{(1)}\left(x_{2}\right) \rho_{N}^{(1)}\left(x_{3}\right) \mathrm{d} x_{2} \mathrm{~d} x_{3} \mathrm{~d} Z_{1} \\
& +o\left(\frac{1}{N}\right) .
\end{aligned}
$$

By the change of variable stated in Lemma 5 and applied to $H=K^{\prime}, \rho=\rho_{N}^{(1)}, x=x_{2}$, and $r=\left|x_{1}-x_{2}\right|$ we have

$$
\begin{aligned}
& \int_{x_{3} \notin B\left(x_{2},\left|x_{1}-x_{2}\right|\right)} K^{\prime}\left(M_{\rho_{N}^{(1)}}\left(x_{2},\left|x_{2}-x_{3}\right|\right)\right) \rho_{N}^{(1)}\left(x_{3}\right) \mathrm{d} x_{3} \\
& =K(1)-K\left(M_{\rho_{N}^{(1)}}\left(x_{2},\left|x_{1}-x_{2}\right|\right) .\right.
\end{aligned}
$$

Inserting this in (1) we obtain

$$
\begin{aligned}
N \times(1)= & K(1) \int \phi\left(Z_{1}\right) f_{N}^{(1)}\left(Z_{1}\right) \mathrm{d} Z_{1} \int \rho_{N}^{(1)}\left(x_{2}\right) \mathrm{d} x_{2} \\
& -\int \phi\left(Z_{1}\right) f_{N}^{(1)}\left(Z_{1}\right) \rho_{N}^{(1)}\left(x_{2}\right) K\left(M_{\rho_{N}^{(1)}}\left(x_{2},\left|x_{1}-x_{2}\right|\right) \mathrm{d} x_{2} \mathrm{~d} Z_{1}+o(1)\right. \\
= & K(1) \int \phi\left(Z_{1}\right) f_{N}^{(1)}\left(Z_{1}\right) \mathrm{d} Z_{1} \\
& -\int \phi\left(Z_{1}\right) f_{N}^{(1)}\left(Z_{1}\right) \rho_{N}^{(1)}\left(x_{2}\right) K\left(M_{\rho_{N}^{(1)}}\left(x_{2},\left|x_{1}-x_{2}\right|\right) \mathrm{d} x_{2} \mathrm{~d} Z_{1}+o(1) .\right.
\end{aligned}
$$


- Using again the change of variable result of Lemma 5 together with integration by parts, we compute:

$$
\begin{aligned}
\int & \left(K(p)+\frac{1-2 p}{N} K^{\prime}(p)+\frac{p(1-p)}{2 N} K^{\prime \prime}(p)\right) \rho_{N}^{(1)}\left(x_{3}\right) \mathrm{d} x_{3} \\
& =\int_{0}^{1}\left(K(\tilde{p})+\frac{1-2 \tilde{p}}{N} K^{\prime}(\tilde{p})+\frac{\tilde{p}(1-\tilde{p})}{2 N} K^{\prime \prime}(\tilde{p})\right) d \tilde{p} \\
& =1+\frac{1}{N}-\frac{K(0)+K(1)}{2 N},
\end{aligned}
$$

where $p=M_{\rho_{N}^{(1)}}\left(x_{2},\left|x_{2}-x_{3}\right|\right)$. From this and Lemma 3, we deduce:

$$
\text { (2) }=\left(1+\frac{1}{N}-\frac{K(0)+K(1)}{2 N}\right) \int \phi\left(Z_{1}\right) f_{N}^{(1)}\left(Z_{1}\right) \mathrm{d} Z_{1}+o\left(\frac{1}{N}\right) .
$$

- Combining the two terms (8) and (9), we obtain the result stated in Lemma 4.

We are now ready to prove Proposition 5

Proof of Proposition 5 The proof is divided in two main steps.

- We first have

$$
\begin{aligned}
S^{N}(K) & =\frac{1}{N-1} \sum_{k=1}^{N-1} K\left(\frac{k}{N-1}\right) \\
& =\frac{K(1)-K(0)}{2(N-1)}+\frac{1}{N-1}\left(\frac{K(0)+K(1)}{2}+\sum_{k=1}^{N-2} K\left(\frac{k}{N-1}\right)\right) .
\end{aligned}
$$

In the second term of this expression, we recognise the approximation of $\int_{0}^{1} K(s) \mathrm{d} s$ by the trapezoidal rule. As the trapezoidal rule is second order, it leads to

$$
\begin{aligned}
S^{N}(K) & =\frac{K(1)-K(0)}{2(N-1)}+\int_{0}^{1} K(s) \mathrm{d} s+o\left(\frac{1}{N}\right) \\
& =\frac{K(1)-K(0)}{2 N}+1+o\left(\frac{1}{N}\right) .
\end{aligned}
$$

As a consequence

$$
\begin{aligned}
\frac{N-2}{S^{N}(K)} & =N \frac{1-2 / N}{1+(K(1)-K(0)) / 2 N+o(1 / N)} \\
& =N-\frac{K(1)-K(0)}{2}-2+o(1) .
\end{aligned}
$$

- Now collecting the estimate of Corollary 4 and (10) we obtain

$$
\begin{aligned}
\left(C^{N}\right)= & \frac{N-2}{S^{N}(K)}[(1)+(2)] \\
= & \left(N-\frac{K(1)-K(0)}{2}-2\right) \int \phi\left(Z_{1}\right) f_{N}^{(1)}\left(Z_{1}\right) \mathrm{d} Z_{1} \\
& +\left(1+\frac{K(1)-K(0)}{2}\right) \int \phi\left(Z_{1}\right) f_{N}^{(1)}\left(Z_{1}\right) \mathrm{d} Z_{1} \\
& -\int \phi\left(Z_{1}\right) f_{N}^{(1)}\left(Z_{1}\right) \rho_{N}^{(1)}\left(x_{2}\right) K\left(M_{\rho_{N}^{(1)}}\left(x_{2},\left|x_{1}-x_{2}\right|\right) \mathrm{d} x_{2} \mathrm{~d} Z_{1}+o(1) .\right.
\end{aligned}
$$


And as

$$
\left(D^{N}\right)=-N \int \phi\left(Z_{1}\right) f_{N}^{(1)}\left(Z_{1}\right) \mathrm{d} Z_{1}
$$

we obtain the statement of Proposition 5.

\subsection{Proof of Theorem 1}

We have to pass to the limit in (4). By Propositions 4 and 5 we have

$$
\begin{aligned}
\partial_{t} \int & f_{N}^{(1)}\left(Z_{1}\right) \phi\left(Z_{1}\right) \mathrm{d} Z_{1}-\sum_{i=1}^{N} \int f_{N}^{(1)}(\mathbf{Z})\left(v_{i} \cdot \nabla_{x_{i}}\right) \phi(\mathbf{Z}) \mathrm{d} \mathbf{Z} \\
= & \left(\frac{1}{S^{N}(K)}-1\right) \int \phi\left(Z_{1}\right) f_{N}^{(1)}\left(Z_{1}\right) K\left(M_{\rho_{N}^{(1)}}\left(x_{2},\left|x_{1}-x_{2}\right|\right)\right) \rho_{N}^{(1)}\left(x_{2}\right) \mathrm{d} Z_{1} \mathrm{~d} x_{2} \\
& +\frac{1}{S^{N}(K)} \int \phi\left(x_{1}, v_{2}\right) \rho_{N}^{(1)}\left(Z_{1}\right) f_{N}^{(1)}\left(Z_{2}\right) K\left(M_{\rho_{N}^{(1)}}\left(x_{1},\left|x_{1}-x_{2}\right|\right)\right) \mathrm{d} x_{1} \mathrm{~d} Z_{2} \\
& -\int \phi\left(Z_{1}\right) f_{N}^{(1)}\left(Z_{1}\right) \mathrm{d} Z_{1}+o(1) .
\end{aligned}
$$

As $N$ goes to $\infty$, the second line goes to 0 since $S^{N}(K)$ is the Riemann sum approximation of $\int_{0}^{1} K(r) \mathrm{d} r=1$. The convergence in the other terms is formally obvious and leads to the stated result.

\section{Discussion}

\subsection{Large-Time Behaviour}

Consider a function homogeneous in space $(t, x, v) \mapsto G(t, v)$. Since $\int K=1$, by Lemma 5 , we get

$$
\frac{\partial G}{\partial t}(v)=-v \cdot \nabla_{x} G(v)+G(v) \int K\left(M_{\rho}\left(x,\left|x^{\prime}-x\right|\right)\right) \mathrm{d} x^{\prime}-G(v)=0 .
$$

Hence any function homogeneous in space $(t, x, v) \mapsto G(t, v)$ is a stationary solution. Moreover, on a periodic spatial domain, we can expect that any solution converges at largetimes toward a function of this type. The proof of such a claim is left to future work.

\subsection{Discrete Versus Continuous Approach}

We can wonder if the large-time and large number of particles limits permute. It does not seem the case. Indeed, the number of distinct velocities decreases when there is a finite number of particles while, as discussed in the previous section, the distribution of velocities remains constant in time in the case of a continuum of particles.

In the case of a finite number of particles the consensus in the direction the particles adopt is longer and longer to obtain, see Figs. 1, 2 and 3. 

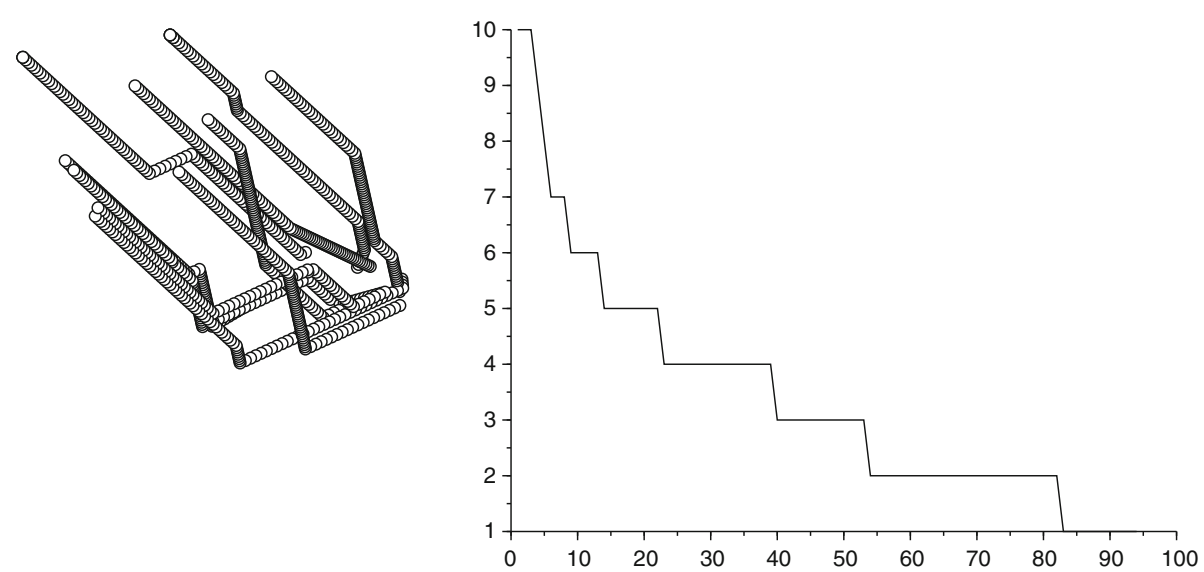

Fig. 1 Trajectories of the particles on the left, variance and number of different speed as functions of time in the case of 10 particles taken randomly in $[-10,10]$ for the position and for the speed
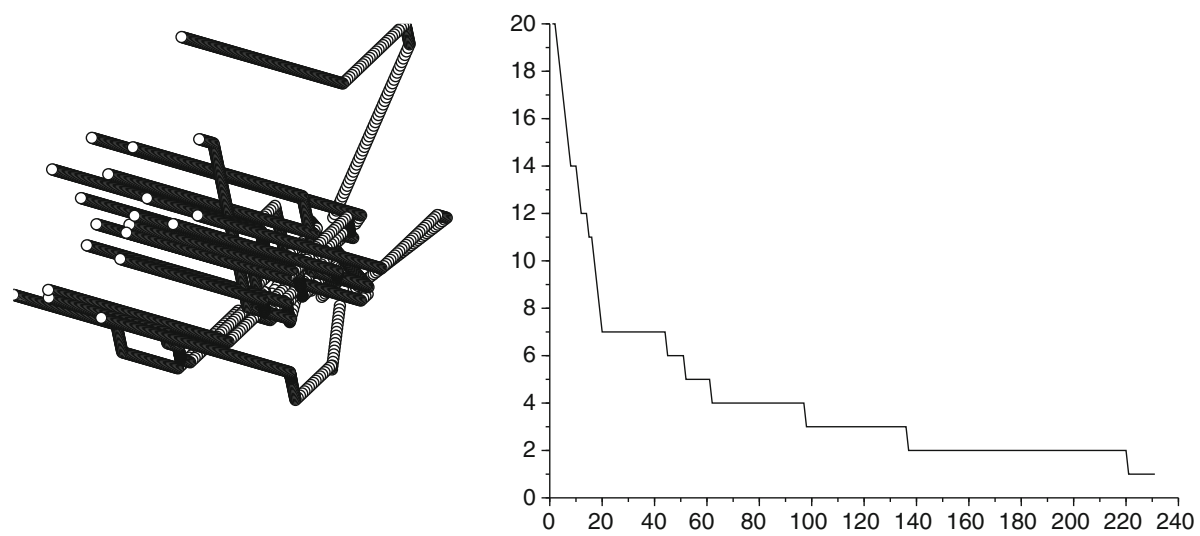

Fig. 2 Trajectories of the particles on the left, variance and number of different speed as functions of time in the case of 20 particles taken randomly in $[-10,10]$ for the position and for the speed
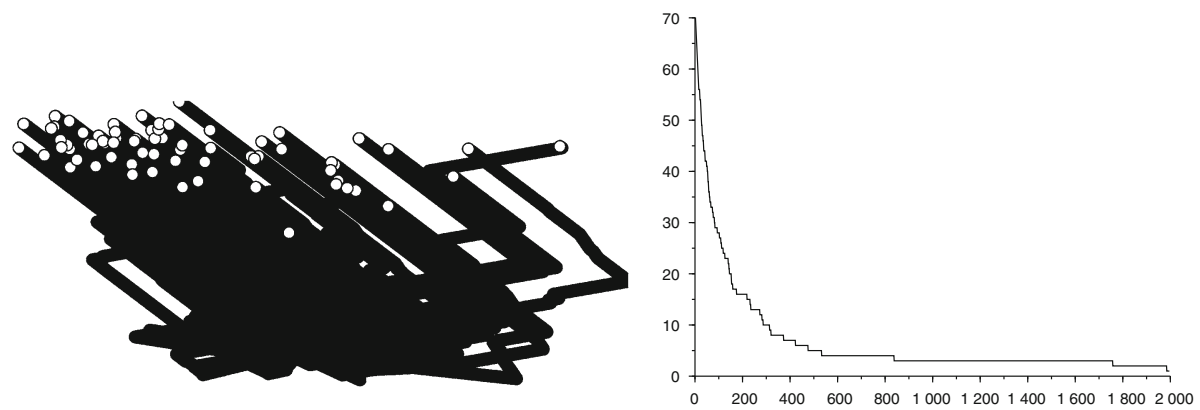

Fig. 3 Trajectories of the particles on the left, variance and number of different speed as functions of time in the case of 70 particles taken randomly in $[-10,10]$ for the position and for the speed 


\section{Conclusion}

In this paper, we have investigated a system of particles interacting through leader following interactions where the choice of the leader is determined by a topological rule. Under a propagation of chaos assumption, we have shown that the large system size limit is described by a spatially nonlocal kinetic model of Boltzmann type. This result heavily relies on approximation properties of Bernstein polynomials. Obviously, the very simple leader following model considered in this paper offers many directions of complexification leading to biologically or socially more realistic rules. An example could be the introduction of some noise, e.g. the velocity after the interaction would be randomly selected according to a probability law centred around the leader velocity. One could also think of the two particles joining their average velocity up to some noise, in the spirit of [2]. Finally, binary interactions with the closest neighbour could also be investigated.

Acknowledgments This work has been supported by the Engineering and Physical Sciences Research Council (EPSRC) under grant ref: EP/M006883/1, by the Agence Nationale pour la Recherche (ANR) under grant MOTIMO (ANR-11-MONU-009-01) and by the National Science Foundation (NSF) under grant RNMS1107444 (KI-Net). PD is on leave from CNRS, Institut de Mathématiques de Toulouse, France. PD gratefully acknowledges support from the Royal Society and the Wolfson foundation through a Royal Society Wolfson Research Merit Award.

Open Access This article is distributed under the terms of the Creative Commons Attribution 4.0 International License (http://creativecommons.org/licenses/by/4.0/), which permits unrestricted use, distribution, and reproduction in any medium, provided you give appropriate credit to the original author(s) and the source, provide a link to the Creative Commons license, and indicate if changes were made.

\section{Appendix: Fundamental Lemma}

Lemma 5 For any $H$,

$$
I(x ; r):=\int_{B_{r}(x)} H\left(M_{\rho}\left(x,\left|x^{\prime}-x\right|\right)\right) \rho\left(x^{\prime}\right) \mathrm{d} x^{\prime}=\int_{0}^{M_{\rho}(x, r)} H(p) \mathrm{d} p .
$$

Proof First note that since

$$
M_{\rho}(x, s)=\int_{\tilde{s}<s} \int_{\omega \in \mathbb{S}^{n-1}} \rho(x+\tilde{s} \omega) \tilde{s}^{n-1} \mathrm{~d} \tilde{s} \mathrm{~d} \omega
$$

we have

$$
\frac{\mathrm{d}}{\mathrm{d} s} M_{\rho}(x, s)=\int_{\omega \in \mathbb{S}^{n-1}} \rho(x+s \omega) s^{n-1} \mathrm{~d} \omega .
$$

Using the polar change of variables,

$$
\left|x^{\prime}-x\right|=: s \quad \frac{x^{\prime}-x}{\left|x^{\prime}-x\right|}=: \omega,
$$

we have

$$
\begin{aligned}
I(x ; r) & =\int_{s<r} \int_{\omega \in \mathbb{S}^{n-1}} \rho(x+s \omega) H\left(M_{\rho}(x, s)\right) s^{n-1} \mathrm{~d} s \mathrm{~d} \omega \\
& =\int_{s<r} \frac{\mathrm{d}}{\mathrm{d} s} M_{\rho}(x, s) H\left(M_{\rho}(x, s)\right) \mathrm{d} s .
\end{aligned}
$$

Setting $p=M_{\rho}(x, s)$, so that $\mathrm{d} p=\frac{\mathrm{d}}{\mathrm{d} s} M_{\rho}(x, s) \mathrm{d} s$, we obtain the stated result. 


\section{References}

1. Ballerini, M., Cabibbo, N., Candelier, R., Cavagna, A., Cisbani, E., Giardina, I., Lecomte, V., Orlandi, A., Parisi, G., Procaccini, A., et al.: Interaction ruling animal collective behavior depends on topological rather than metric distance: evidence from a field study. Proc. Natl. Acad. Sci. 105(4), 1232-1237 (2008)

2. Bertin, E., Droz, M., Grégoire, C.: Boltzmann and hydrodynamic description for self-propelled particles. Phys. Rev. E 74, 022101 (2006)

3. Bertozzi, A.L., Laurent, T.: Finite-time blow-up of solutions of an aggregation equation in $\mathrm{r} n$. Commun. Math. Phys. 274(3), 717-735 (2007)

4. Bertozzi, A.L., Carrillo, J.A., Laurent, T.: Blow-up in multidimensional aggregation equations with mildly singular interaction kernels. Nonlinearity 22(3), 683 (2009)

5. Bode, N.W., Franks, D.W., Wood, A.J.: Limited interactions in flocks: relating model simulations to empirical data. J. R. Soc. Interface 8, 301-304 (2010)

6. Brenier, Y.: L2 formulation of multidimensional scalar conservation laws. Arch. Ration. Mech. Anal. 193(1), 1-19 (2009)

7. Camperi, M., Cavagna, A., Giardina, I., Parisi, G., Silvestri, E.: Spatially balanced topological interaction grants optimal cohesion in flocking models. Interface Focus 2(6), 715-725 (2012)

8. Carlen, E., Chatelin, R., Degond, P., Wennberg, B.: Kinetic hierarchy and propagation of chaos in biological swarm models. Physica D 260, 90-111 (2013)

9. Carlen, E., Degond, P., Wennberg, B.: Kinetic limits for pair-interaction driven master equations and biological swarm models. Math. Models Methods Appl. Sci. 23(07), 1339-1376 (2013)

10. Cavagna, A., Giardina, I., Orlandi, A., Parisi, G., Procaccini, A., Viale, M., Zdravkovic, V.: The starflag handbook on collective animal behaviour: 1. Empirical methods. Animal Behav. 76(1), 217-236 (2008)

11. Cucker, F., Smale, S.: Emergent behavior in flocks. IEEE Trans. Autom. Control 52(5), 852-862 (2007)

12. Dewsbury, D.A.: Dominance rank, copulatory behavior, and differential reproduction. Q. Rev. Biol. 57, 135-159 (1982)

13. Ginelli, F., Chaté, H.: Relevance of metric-free interactions in flocking phenomena. Phys. Rev. Lett. 105(16), 168103 (2010)

14. Harsanyi, J.C.: Cardinal Welfare, Individualistic Ethics, and Interpersonal Comparisons of Utility. Springer, Amsterdam (1976)

15. Haskovec, J.: Flocking dynamics and mean-field limit in the cucker-smale-type model with topological interactions. Physica D 261, 42-51 (2013)

16. Horrocks, J., Hunte, W.: Maternal rank and offspring rank in vervet monkeys: an appraisal of the mechanisms of rank acquisition. Animal Behav. 31(3), 772-782 (1983)

17. Lazear, E.P., Rosen, S.: Rank-order tournaments as optimum labor contracts. J. Political Econ. 89(5), 841-864 (1981)

18. Lorentz, G.G.: Bernstein Polynomials. American Mathematical Society, London (2012)

19. Niizato, T., Gunji, Y.P.: Metric-topological interaction model of collective behavior. Ecol. Model. 222(17), 3041-3049 (2011)

20. Vicsek, T., Czirók, A., Ben-Jacob, E., Cohen, I., Shochet, O.: Novel type of phase transition in a system of self-driven particles. Phys. Rev. Lett. 75(6), 1226 (1995) 